\title{
Association between Microsatellite Polymorphism and Body Size Traits in Sonid Bactrian Camels
}

\author{
Jun Yan Bai ${ }^{1 *}$, Ren Tao Di Wu ${ }^{2}$, Qiang Zhang ${ }^{2}$, Di Bao ${ }^{2}$, Le Ma Dao ${ }^{2}$ and \\ Xing Hua Tian ${ }^{3}$ \\ ${ }^{1}$ College of Animal Science and Technology, Henan University of Science and \\ Technology, Luoyang 471023, China, \\ ${ }^{2}$ Institute of Animal, Alxa of Inner Mongolia, Bayanhaote 750306, China \\ ${ }^{3}$ School of Life Sciences, Henan Universiry, Kaifeng 475000, China
}

\section{A B S T R A C T}

In this study, 17 microsatellite markers were used to analyze the genetic diversity of Sonid bactrian camels, and the correlation between microsatellite markers and their body size was analyzed to find microsatellite markers associated with the body size traits of Sonid bactrian camels, so as to provide reference for marker-assisted selection of Sonid bactrian camels. The results showed that 64 alleles were detected in 17 microsatellite markers. The average number of alleles was 3.7647 , the average heterozygosity was 0.6205 , and the average polymorphism information content was 0.5602 . It showed that the population of Sonid bactrian camel had high genetic diversity. Among 17 microsatellite markers, 4 microsatellites were found to be associated with body size traits of Sonid bactrian camels. The body length of AB genotype labeled LCA33 was significantly higher than that of AA genotype $(P<0.05)$, and the chest circumference, tube circumference and body weight of $\mathrm{AB}$ genotype were significantly higher than those of AA genotype and AC genotype $(P<0.05)$. The chest circumference and body weight of A A genotype marked LCA90 were significantly higher than those of AC genotype and $\mathrm{BB}$ genotype $(P<0.05)$. The body length of $\mathrm{AB}$ genotype marked CMS36 was significantly higher than that of AA genotype and BB genotype $(P<0.05)$. The body weight of CE genotype marked YWLL44 was significantly higher than that of AC genotype, $\mathrm{BD}$ genotype and $\mathrm{BE}$ genotype $(P<0.05)$, the circumference of $\mathrm{CE}$ genotype was significantly higher than that of AC genotype $(P<0.05)$, and the chest circumference of CE genotype was significantly higher than that of AC genotype and BD genotype $(P<0.05)$. The other 13 microsatellite markers had no significant effect on the body size of Sonid bactrian camels.
\end{abstract}

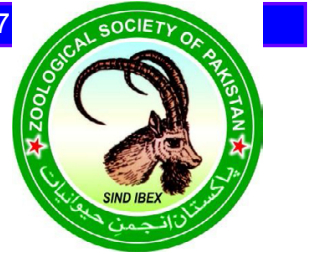

Article Information
Received 05 September 2019
Revised 22 September 2019
Accepted 01 October 2019
Available online 12 February 2021
Authors' Contribution
JYB conceived and designed the study
and conducted the lab work. RTDW
and QZ analyzed the data and wrote
the article. DB and LMD helped in
sampling. XHT helped in analysis
of data.
Key words
Sonid bactrian camel, Microsatellite
markers, Genetic diversity, Body size
traits, Association analysis

\section{INTRODUCTION}

$\mathrm{B}$ actrian camels, which are special livestock species with double back humps and tall body and adapting to desert and semi-desert regions, mainly live in deserts, and their range of activity is mainly located in western and northern Asia. The region where bactrian camels live is very narrow and it is only restricted to arid areas, so they are precious livestock species in the world. In recent years, domestic and foreign researches regarding hair characteristics (Wuren et al., 2017), dairy products (Zhang et al., 2016), blood physiological properties (Bai et al., 2015), genetic diversity (Hedayat-Evrigh et al., 2018; Banerjee et al., 2012) and organization structure (Ye et al., 2014a, 2014b) of bactrian camels have achieved progress.

Microsatellite markers have been extensively applied to genetic diversity studies of cow ( $\mathrm{Ni}$ et al., 2018), sheep (Bai et al., 2015) and poultry (Bai et al., 2016a, 2016b, 2016c, 2017) by virtue of high abundance,

\footnotetext{
Corresponding author: junyanbai@163.com 0030-9923/2021/0002-0515 \$ 9.00/0

Copyright 2021 Zoological Society of Pakistan
}

good repeatability, co-dominance marker and selective neutrality, It is also widely used in plant genetic diversity research (Yang et al., 2013; Li et al.,2017; Guo et al., 2018). Genetic diversity of 17 microsatellite markers in the Sonid Bactrian camel population was analyzed in this study, association analysis of these microsatellite markers with body weight and body size and other traits was conducted, and microsatellite markers associated with body size traits of Sonid bactrian camels was found out so as to provide a reference for marker assisted selection, protection and rational utilization of Sonid bactrian camels.

\section{MATERIALS AND METHODS}

\section{Test material}

Random collection of 40 Sonid bactrian camels, blood was taken from jugular vein $(10 \mathrm{ml}$ from each bactrian camel), ACD anticoagulant was added for anticoagulation, blood DNA was extracted using the whole-blood genomic DNA extraction kit method from Beijing Dingguo Changsheng, and it was preserved under $-20^{\circ} \mathrm{C}$. Seventeen microsatellite markers with high polymorphism were screened (Evdotchenko et al., 2003; Prasad et al., 2014), 
Table I. Microsatellite marker information.

\begin{tabular}{llll}
\hline Name & Primer sequence & $\begin{array}{c}\text { Fragment } \\
\text { size (bp) }\end{array}$ & $\begin{array}{l}\text { Annealing } \\
\text { temperature }\end{array}$ \\
\hline CVRL101 & F:GAAGAGGTTGGGGCACTAC; R:CAGGCAGATATCCATTGAA & $188-253$ & 60 \\
CMS15 & F:AAAACTAAAGCCAGAAAGGCAAA; R:TTTTTCCAGATCTTGCACCAC & $81-121$ & 58 \\
CMS18 & F:GAACGACCCTTGAAGACGAA; R:AGCAGCTGGTTTTAGGTCCA & $144-166$ & 55 \\
CMS36 & F:TGCTTTCCAGTTGTTTGCTG; R:GCAAGGTGGTGTTGGAGATT & $195-227$ & 55 \\
CMS104 & F:CACTTAGGTCCCTGGGCTTT; R:GCATTCTCTTGCATCGTGTG & $75-101$ & 55 \\
LCA33 & F:GAGCACAGGGAAGGATATTCA; R:ACAGCAAAGTGATTCCATAATACA & $122-167$ & 55 \\
LCA37 & F:TAATTACCTCCCCCACCACA; R:TGGACCCAGGACTTGAAATG & $143-183$ & 55 \\
LCA63 & F:TTACCCAGTCCTTCGTGGG; R:GGAACCTCGTGGTTATGGAA & $213-233$ & 54 \\
LCA66 & F:GTGCAGCGTCCAAATAGTCA; R:CCAGCATCGTCCAGTATTCA & $212-241$ & 55 \\
LCA71 & F:CCGTATCTATCTATACACACACACACA; R:TCACCCTCCTCCTATTTTGG & $127-167$ & 55 \\
LCA82 & F:CGTGACACCAGGCTAAGTGA; R:TTTCAGATGGTAGCTTTAAAAATTG & $85-129$ & 55 \\
LCA90 & F:TATAACCCTGGTCTCGCCAA; R:CCAAGTAGTATTCCATTATGCG & $237-248$ & 55 \\
VOLP08 & F:CCATTCACCCCATCTCTC; R:TCGCCAGTGACCTTATTTAGA & $142 ~ 172$ & 53.3 \\
VOLP32 & F:GTGATCGGAATGGCTTGAAA; R:CAGCGAGCACCTGAAAGAA & $147-203$ & 53.3 \\
YWLL29 & F:GAAGGCAGGAGAAAAGGTAG; R:CAGAGGCTTAATAACTTGCAG & $200-228$ & 55 \\
YWLL36 & F:CAAATGTTGTAAAATTGCACAGAAC; R:TTCAGGTTTGCCTAGTTTTAACTGT & $149-189$ & 55
\end{tabular}

and the sequence of primers was shown in Table I. The primers were synthesized by Shanghai Shenggong Bioengineering Technology Service Co., Ltd.

\section{PCR amplification}

Pre-denaturation at 94 for $4 \mathrm{~min}$, then denaturation at 94 for $40 \mathrm{~s}$, annealing at 60 for $1 \mathrm{~min}$, annealing at 72 for 20 seconds, denaturation, annealing and elongation were carried out for 35 cycles, then elongation at 72 and finally the reaction was completed and cooled and preserved at $4 \mathrm{C}$.
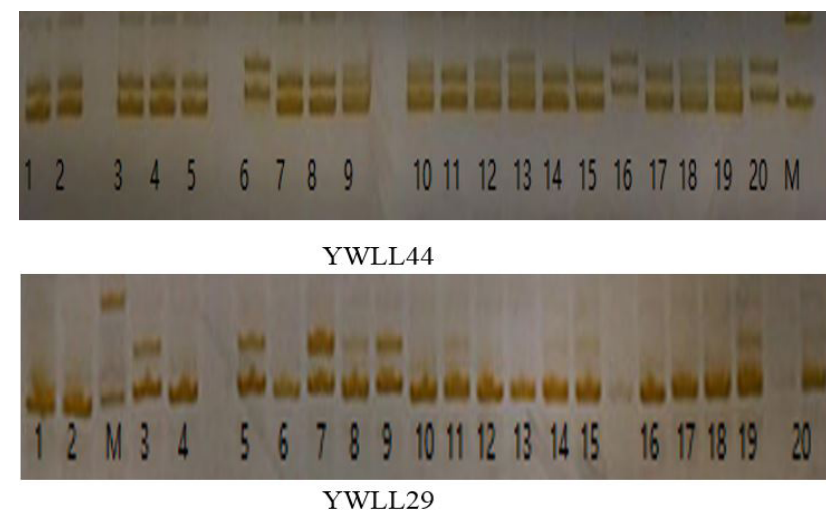

Fig. 1. Polymorphism of microsatellite markers YWLL44 and YWLL29 in Sonid bactrian camels.
Table II. Allele frequencies of microsatellite markers.

\begin{tabular}{llllll}
\hline Microsatellite & \multicolumn{5}{c}{ Alleles } \\
\cline { 2 - 6 } markers & $\mathbf{A}$ & B & C & D & E \\
\hline LCA33 & 0.6000 & 0.0875 & 0.2500 & 0.0625 & \\
LCA37 & 0.4750 & 0.5000 & 0.0250 & & \\
LCA63 & 0.4250 & 0.1375 & 0.3625 & 0.0750 & \\
LCA66 & 0.1625 & 0.3375 & 0.1625 & 0.3375 & \\
LCA71 & 0.0625 & 0.4625 & 0.4750 & & \\
LCA82 & 0.4500 & 0.0500 & 0.4500 & 0.0500 & \\
LCA90 & 0.3500 & 0.2750 & 0.1750 & 0.2000 & \\
CMS15 & 0.0375 & 0.2875 & 0.4625 & 0.2125 & \\
CMS18 & 0.5000 & 0.2875 & 0.1875 & 0.0250 & \\
CMS36 & 0.5875 & 0.4125 & & & \\
CMS104 & 0.5125 & 0.3750 & 0.1125 & & \\
CVRL101 & 0.1375 & 0.3000 & 0.2000 & 0.2375 & 0.1250 \\
YWLL29 & 0.6625 & 0.1125 & 0.2250 & & \\
YWLL36 & & 0.0250 & 0.4750 & 0.0250 & 0.4750 \\
YWLL44 & 0.0250 & 0.4375 & 0.0625 & 0.2625 & 0.2125 \\
VOLP08 & 0.2250 & 0.2750 & 0.2250 & 0.2750 & \\
VOLP32 & 0.2875 & 0.2125 & 0.2875 & 0.2125 & \\
\hline
\end{tabular}

The amplified product $5 \mu 1$ was detected by $2 \%$ agarose electrophoresis at $120 \mathrm{~V}$ for $30 \mathrm{~min}$. After electrophoresis, the results were observed under ultraviolet light and photographed on the basis of DNA marker D2000. 
Table III. Polymorphic information of microsatellite markers.

\begin{tabular}{lllllllll}
\hline $\begin{array}{l}\text { Microsatellite } \\
\text { markers }\end{array}$ & Na & Ne & O_Hom & O_Het & E_Hom & E_Het & Ave_Het & PIC \\
\hline LCA33 & 4.0000 & 2.3038 & 0.2500 & 0.7500 & 0.4269 & 0.5731 & 0.5356 & 0.5111 \\
LCA37 & 3.0000 & 2.0997 & 0.0000 & 1.0000 & 0.4696 & 0.5304 & 0.5248 & 0.4103 \\
LCA63 & 4.0000 & 2.9712 & 0.0000 & 1.0000 & 0.3282 & 0.6718 & 0.6756 & 0.6004 \\
LCA66 & 4.0000 & 3.5635 & 0.0000 & 1.0000 & 0.2715 & 0.7285 & 0.7330 & 0.6680 \\
LCA71 & 3.0000 & 2.2551 & 0.0500 & 0.9500 & 0.4364 & 0.5636 & 0.5727 & 0.4566 \\
LCA82 & 4.0000 & 2.4390 & 0.0000 & 1.0000 & 0.4025 & 0.5975 & 0.6045 & 0.5039 \\
LCA90 & 4.0000 & 3.7209 & 0.2500 & 0.7500 & 0.2595 & 0.7405 & 0.7270 & 0.6823 \\
CMS15 & 4.0000 & 2.9144 & 0.0000 & 1.0000 & 0.3348 & 0.6652 & 0.6782 & 0.5938 \\
CMS18 & 4.0000 & 2.7142 & 0.0000 & 1.0000 & 0.3604 & 0.6396 & 0.6292 & 0.5664 \\
CMS36 & 2.0000 & 1.9406 & 0.8750 & 0.1250 & 0.5092 & 0.4908 & 0.3795 & 0.3672 \\
CMS104 & 3.0000 & 2.4042 & 0.1250 & 0.8750 & 0.4085 & 0.5915 & 0.5728 & 0.5000 \\
CVRL101 & 5.0000 & 4.5262 & 0.0000 & 1.0000 & 0.2111 & 0.7889 & 0.7464 & 0.7437 \\
YWLL29 & 3.0000 & 1.9913 & 0.5500 & 0.4500 & 0.4959 & 0.5041 & 0.4025 & 0.4410 \\
YWLL36 & 4.0000 & 2.2099 & 0.0000 & 1.0000 & 0.4456 & 0.5544 & 0.5993 & 0.4446 \\
YWLL44 & 5.0000 & 3.2258 & 0.0000 & 1.0000 & 0.3013 & 0.6987 & 0.6963 & 0.6373 \\
VOLP08 & 4.0000 & 3.9604 & 0.0000 & 1.0000 & 0.2430 & 0.7570 & 0.7413 & 0.7003 \\
VOLP32 & 4.0000 & 3.9120 & 0.0000 & 1.0000 & 0.2462 & 0.7538 & 0.7298 & 0.6968 \\
Mean & 3.7647 & 2.8913 & 0.1235 & 0.8765 & 0.3618 & 0.6382 & 0.6205 & 0.5602 \\
\hline
\end{tabular}

\section{SSCP}

$15 \%$ non denaturing polyacrylamide gels were used to detect the products, silver nitrate dyeing method is used for dyeing, mainly through fixation, oxidation, dyeing, color rendering, photography and other links.

\section{Statistical analysis}

Popgene32 software was used to calculate numbers of effective alleles, allele frequencies and heterozygosity of microsatellites. SPSS software was used to conduct association analysis of microsatellites with body weight, and the following was the analytical model: $y_{i j k l}=\mu+S_{i}+M_{j}+G_{k}+e_{i j k l}$, where $y_{i j k l}$ is trait phenotypic value, $\mu$ is ensemble average and $S_{i}$ is the ${ }_{i}$ (th) sex effect; $M_{j}$ is the $j$ (th) age effect; $G_{k}$ is the $k$ (th) genotype effect; $e_{i j k l}{ }^{j}$ is residual error effect.

\section{RESULTS AND DISCUSSION}

\section{Polymorphism detection of microsatellite markers}

The polyacrylamide gel electrophoresis results of PCR products of some markers are presented in Figure 1. The allele frequencies of microsatellite markers in Sonid bactrian camel population are shown in Table II. The maximum number of alleles detected by CVRL101 is 5 , the minimum number of alleles detected by CMS36 is 2 , the number of alleles detected by other markers is between 3 and 4 .

The polymorphism of microsatellite markers can be seen in Table III, 64 alleles were detected in 17 microsatellite markers. The average number of alleles was 3.7647 , the average heterozygosity was 0.6205 , and the average polymorphism information content was 0.5602 . It can be seen that the Sonid bactrian camel has high genetic diversity. The study carried out by Gao et al. (2009) showed that the average polymorphic information content value of microsatellite markers detected in hundreds of bactrian camels from 13 areas of China and Mongolia was 0.5414. Tian et al. (2012) studied the genetic diversity of bactrian camels from 6 places of Xinjiang by microsatellite markers, and showed that all of the used microsatellite markers presented high polymorphism, with polymorphic information content values ranging from 0.6099 to 0.6551 . Vijh et al. (2007) conducted a microsatellite marker analysis of 4 dromedary species in India, and results indicated that a large number of alleles were under a low frequency. So the polymorphic level of microsatellite markers in this study was moderate, intermediate between Gao et al. (2009) and Tian et al. (2012). 
Table IV. Association between microsatellite markers and body size of Sonid bactrian camels.

\begin{tabular}{|c|c|c|c|c|c|c|}
\hline Name & Genotype & Body height $/ \mathrm{cm}$ & Body length/cm & Bust/cm & Round tube $/ \mathrm{cm}$ & Weight/ kg \\
\hline \multirow[t]{5}{*}{ LCA33 } & AA & $161.000 \pm 02.0330^{\mathrm{a}}$ & $139.6000 \pm 2.9257^{b}$ & $201.6000 \pm 3.3373^{b}$ & $16.8000 \pm 0.2494^{b}$ & $445.2900 \pm 17.6812^{b}$ \\
\hline & $\mathrm{AB}$ & $168.2000 \pm 2.7459^{\mathrm{a}}$ & $154.0000 \pm 3.3015^{\mathrm{a}}$ & $225.4000 \pm 4.2614^{\mathrm{a}}$ & $18.4000 \pm 0.5099^{\mathrm{a}}$ & $568.9090 \pm 22.5901^{\mathrm{a}}$ \\
\hline & $\mathrm{AC}$ & $163.3889 \pm 2.2854^{\mathrm{a}}$ & $145.8889 \pm 2.5112^{\mathrm{ab}}$ & $211.4444 \pm 4.2994^{\mathrm{ab}}$ & $17.2778 \pm 0.3110^{\mathrm{ab}}$ & $498.6865 \pm 22.2293^{\mathrm{ab}}$ \\
\hline & $\mathrm{AD}$ & $165.8000 \pm 0.9695^{\mathrm{a}}$ & $146.4000 \pm 2.5416^{\mathrm{ab}}$ & $218.6000 \pm 5.9883^{\mathrm{ab}}$ & $17.2000 \pm 0.3741^{\mathrm{ab}}$ & $521.3083 \pm 26.7245^{\mathrm{ab}}$ \\
\hline & $\mathrm{BC}$ & $157.5000 \pm 7.5000^{\mathrm{a}}$ & $141.0000 \pm 9.0000^{\mathrm{ab}}$ & $198.5000 \pm 6.5000^{\mathrm{b}}$ & $16.5000 \pm 0.5000^{\mathrm{b}}$ & $437.5177 \pm 41.8083^{b}$ \\
\hline \multirow[t]{2}{*}{ LCA37 } & $\mathrm{AB}$ & $163.2105 \pm 1.3303^{\mathrm{a}}$ & $144.8947 \pm 1.6611^{\mathrm{a}}$ & $210.8421 \pm 2.7096^{\mathrm{a}}$ & $17.2895 \pm 0.1919^{\mathrm{a}}$ & $493.0276 \pm 13.8334^{\mathrm{a}}$ \\
\hline & $\mathrm{BC}$ & $167.0000 \pm 2.0000^{\mathrm{a}}$ & $150.0000 \pm 4.0000^{\mathrm{a}}$ & $213.5000 \pm 2.5000^{\mathrm{a}}$ & $16.5000 \pm 0.5000^{\mathrm{a}}$ & $510.1657 \pm 1.2928^{\mathrm{a}}$ \\
\hline \multirow[t]{3}{*}{ LCA63 } & $\mathrm{AB}$ & $164.0000 \pm 2.7928^{\mathrm{a}}$ & $148.0000 \pm 6.2928^{\mathrm{a}}$ & $210.6000 \pm 7.0964^{\mathrm{a}}$ & $17.8000 \pm 0.6633^{\mathrm{a}}$ & $500.1861 \pm 40.2844^{\mathrm{a}}$ \\
\hline & $\mathrm{AC}$ & $163.4483 \pm 1.5604^{\mathrm{a}}$ & $144.9655 \pm 1.7281^{\mathrm{a}}$ & $212.0690 \pm 3.0681^{\mathrm{a}}$ & $17.1379 \pm 0.2089^{\mathrm{a}}$ & $497.0368 \pm 15.3422^{\mathrm{a}}$ \\
\hline & $\mathrm{BD}$ & $162.6667 \pm 3.5839^{\mathrm{a}}$ & $143.6667 \pm 4.7935^{\mathrm{a}}$ & $206.0000 \pm 7.1039^{\mathrm{a}}$ & $17.3333 \pm 0.4944^{\mathrm{a}}$ & $473.3970 \pm 37.8698^{\mathrm{a}}$ \\
\hline \multirow[t]{2}{*}{ LCA66 } & $\mathrm{AC}$ & $165.8462 \pm 2.6234^{\mathrm{a}}$ & $145.1538 \pm 2.9566^{\mathrm{a}}$ & $213.5385 \pm 5.8677^{\mathrm{a}}$ & $17.4615 \pm 0.3859^{\mathrm{a}}$ & $505.7870 \pm 29.4103^{\mathrm{a}}$ \\
\hline & $\mathrm{BD}$ & $162.2222 \pm 1.3788^{\mathrm{a}}$ & $145.1481 \pm 1.9235^{\mathrm{a}}$ & $209.7407 \pm 2.6287^{\mathrm{a}}$ & $17.1481 \pm 0.2046^{\mathrm{a}}$ & $488.1536 \pm 13.7101^{\mathrm{a}}$ \\
\hline \multirow[t]{3}{*}{ LCA71 } & $\mathrm{AC}$ & $161.8000 \pm 4.1641^{\mathrm{a}}$ & $141.4000 \pm 3.6551^{\mathrm{a}}$ & $202.8000 \pm 5.7740^{\mathrm{a}}$ & $16.8000 \pm 0.3741^{\mathrm{a}}$ & $453.7243 \pm 27.2104^{\mathrm{a}}$ \\
\hline & $\mathrm{BB}$ & $162.0000 \pm 5.0000^{\mathrm{a}}$ & $142.5000 \pm 1.5000^{\mathrm{a}}$ & $208.0000 \pm 5.0000^{\mathrm{a}}$ & $17.0000 \pm 0.0000^{\mathrm{a}}$ & $471.2891 \pm 13.4620^{\mathrm{a}}$ \\
\hline & $\mathrm{B}$ & $163.7273 \pm 1.4123^{\mathrm{a}}$ & $145.8788 \pm 1.8431^{\mathrm{a}}$ & $212.3939 \pm 2.9538^{\mathrm{a}}$ & $17.3333 \pm 0.2161^{\mathrm{a}}$ & $501.3388 \pm 15.1859^{a}$ \\
\hline \multirow[t]{2}{*}{ LCA82 } & $\mathrm{AC}$ & $162.9167 \pm 1.2359^{\mathrm{a}}$ & $145.1944 \pm 1.6382^{\mathrm{a}}$ & $210.6944 \pm 2.5054^{\mathrm{a}}$ & $17.2222 \pm 0.1695^{\mathrm{a}}$ & $492.1925 \pm 12.4821^{\mathrm{a}}$ \\
\hline & $\mathrm{BD}$ & $167.7500 \pm 6.5494^{\mathrm{a}}$ & $144.7500 \pm 6.9447^{\mathrm{a}}$ & $213.5000 \pm 14.1745^{\mathrm{a}}$ & $17.5000 \pm 1.1902^{\mathrm{a}}$ & $509.1126 \pm 77.6066^{\mathrm{a}}$ \\
\hline \multirow[t]{4}{*}{ LCA90 } & AA & $166.0000 \pm 1.4960^{\mathrm{a}}$ & $150.1429 \pm 2.9230^{\mathrm{a}}$ & $221.5714 \pm 5.0749^{\mathrm{a}}$ & $17.8571 \pm 0.5084^{\mathrm{a}}$ & $544.7257 \mathrm{a} \pm 27.4807^{\mathrm{a}}$ \\
\hline & $\mathrm{AC}$ & $163.7857 \pm 1.6811^{\mathrm{a}}$ & $144.6429 \pm 2.4147^{\mathrm{a}}$ & $210.5000 \pm 3.4668^{b}$ & $17.2857 \pm 0.1941^{\mathrm{a}}$ & $488.7325 \pm 17.2819^{\mathrm{b}}$ \\
\hline & $\mathrm{BB}$ & $163.6667 \pm 8.6474^{\mathrm{a}}$ & $146.6667 \pm 9.8375^{\text {a }}$ & $207.0000 \pm 9.0185^{b}$ & $17.6667 \pm 0.8819^{\mathrm{a}}$ & $483.2152 \pm 52.1286^{\mathrm{b}}$ \\
\hline & $\mathrm{BD}$ & $161.8750 \pm 2.3977^{\mathrm{a}}$ & $143.1250 \pm 2.6690^{\mathrm{a}}$ & $207.5000 \pm 4.8140^{\mathrm{b}}$ & $16.8750 \pm 0.3275^{\mathrm{a}}$ & $478.1499 \pm 24.5348^{b}$ \\
\hline \multirow[t]{3}{*}{ CMS15 } & $\mathrm{AB}$ & $165.3333 \pm 2.6666^{\mathrm{a}}$ & $146.3333 \pm 4.1766^{\mathrm{a}}$ & $218.3333 \pm 11.2891^{\mathrm{a}}$ & $17.0000 \pm 0.5773^{\mathrm{a}}$ & $522.6098 \pm 51.1865^{\mathrm{a}}$ \\
\hline & $\mathrm{BC}$ & $163.6000 \pm 1.5481^{\mathrm{a}}$ & $144.6500 \pm 1.7877^{\mathrm{a}}$ & $210.2000 \pm 3.1217^{\mathrm{a}}$ & $17.1500 \pm 0.1956^{\mathrm{a}}$ & $487.9703 \pm 14.9405^{\mathrm{a}}$ \\
\hline & $\mathrm{CD}$ & $162.8235 \pm 2.3890^{\mathrm{a}}$ & $145.5294 \pm 3.1096^{\mathrm{a}}$ & $210.5882 \pm 4.5794^{\mathrm{a}}$ & $17.4118 \pm 0.3644^{\mathrm{a}}$ & $495.7731 \pm 24.6920^{\mathrm{a}}$ \\
\hline \multirow[t]{3}{*}{ CMS18 } & $\mathrm{AB}$ & $164.0000 \pm 1.7286^{\mathrm{a}}$ & $144.5652 \pm 1.9819^{\mathrm{a}}$ & $213.1739 \pm 3.6930^{\mathrm{a}}$ & $17.2609 \pm 0.2608^{\mathrm{a}}$ & $500.9518 \pm 18.9254^{\mathrm{a}}$ \\
\hline & $\mathrm{AC}$ & $162.0000 \pm 2.1224^{\mathrm{a}}$ & $145.4667 \pm 3.0282^{\mathrm{a}}$ & $207.2000 \pm 3.8436^{\mathrm{a}}$ & $17.1333 \pm 0.2905^{\mathrm{a}}$ & $480.7806 \pm 20.0080^{\mathrm{a}}$ \\
\hline & $\mathrm{AD}$ & $167.0000 \pm 1.0000^{\mathrm{a}}$ & $149.5000 \pm 0.5000^{\mathrm{a}}$ & $214.0000 \pm 1.0000^{\mathrm{a}}$ & $18.0000 \pm 0.0000^{\mathrm{a}}$ & $510.8888 \pm 2.3896^{\mathrm{a}}$ \\
\hline \multirow[t]{3}{*}{ CMS36 } & $\mathrm{AA}$ & $162.7619 \pm 1.6314^{\mathrm{a}}$ & $144.5714 \pm 1.9130^{\mathrm{b}}$ & $211.7143 \pm 3.4751^{\mathrm{a}}$ & $17.2381 \pm 0.2478^{\mathrm{a}}$ & $494.6097 \pm 17.3440^{\mathrm{a}}$ \\
\hline & $\mathrm{AB}$ & $167.4000 \pm 1.8055^{\mathrm{a}}$ & $154.0000 \pm 4.3243^{\mathrm{a}}$ & $214.6000 \pm 3.0757^{\mathrm{a}}$ & $17.2000 \pm 0.3741^{\mathrm{a}}$ & $525.4455 \pm 16.1662^{\mathrm{a}}$ \\
\hline & BB & $162.9286 \pm 2.6195^{\mathrm{a}}$ & $142.8571 \pm 2.9442^{\mathrm{b}}$ & $208.5714 \pm 5.2043^{\mathrm{a}}$ & $17.2857 \pm 0.3695^{\mathrm{a}}$ & $481.5249 \pm 26.8202^{\mathrm{a}}$ \\
\hline \multirow[t]{4}{*}{ CMS104 } & AA & $166.0000 \pm 3.7859^{\mathrm{a}}$ & $151.6667 \pm 6.1191^{\mathrm{a}}$ & $210.6667 \pm 4.7022^{\mathrm{a}}$ & $17.0000 \pm 0.5773^{\mathrm{a}}$ & $505.8133 \pm 33.0667^{\mathrm{a}}$ \\
\hline & $\mathrm{AB}$ & $163.4615 \pm 1.7639^{a}$ & $144.2308 \pm 2.0140^{\mathrm{a}}$ & $211.8077 \pm 3.6089^{a}$ & $17.3077 \pm 0.2403^{\mathrm{a}}$ & $495.8371 \pm 18.4025^{\mathrm{a}}$ \\
\hline & $\mathrm{AC}$ & $162.0000 \pm 2.2173^{\mathrm{a}}$ & $143.6667 \pm 2.9154^{\mathrm{a}}$ & $208.3333 \pm 4.7900^{\text {a }}$ & $17.1111 \pm 0.4231^{\mathrm{a}}$ & $478.9616 \pm 22.6887^{\mathrm{a}}$ \\
\hline & $\mathrm{BB}$ & $165.0000 \pm 3.0000^{\mathrm{a}}$ & $154.0000 \pm 9.0000^{\mathrm{a}}$ & $212.5000 \pm 2.5000^{\mathrm{a}}$ & $17.5000 \pm 0.5000^{\mathrm{a}}$ & $517.7604 \pm 33.1562^{a}$ \\
\hline \multirow[t]{4}{*}{ CVRL101 } & $\mathrm{AB}$ & $165.6000 \pm 1.0295^{\mathrm{a}}$ & $144.2000 \pm 1.5937^{\mathrm{a}}$ & $217.0000 \pm 5.9916^{\mathrm{a}}$ & $17.0000 \pm 0.0000^{\mathrm{a}}$ & $509.7066 \pm 26.4570^{\mathrm{a}}$ \\
\hline & $\mathrm{AC}$ & $163.1667 \pm 1.7966^{\mathrm{a}}$ & $142.0000 \pm 4.0166^{\mathrm{a}}$ & $207.6667 \pm 7.1305^{\mathrm{a}}$ & $17.0000 \pm 0.4472^{\mathrm{a}}$ & $473.8557 \pm 34.8770^{\mathrm{a}}$ \\
\hline & $\mathrm{BD}$ & $162.8421 \pm 1.5844^{\mathrm{a}}$ & $146.3158 \pm 2.2530^{\mathrm{a}}$ & $211.6842 \pm 3.3113^{\mathrm{a}}$ & $17.3684 \pm 0.2560^{\mathrm{a}}$ & $498.6778 \pm 17.5233^{\mathrm{a}}$ \\
\hline & $\mathrm{CE}$ & $163.5000 \pm 4.1048^{\mathrm{a}}$ & $145.3000 \pm 4.2165^{\mathrm{a}}$ & $208.6000 \pm 6.7019^{\mathrm{a}}$ & $17.3000 \pm 0.5174^{\mathrm{a}}$ & $488.8834 \pm 34.9227^{\mathrm{a}}$ \\
\hline \multirow[t]{3}{*}{ YWLL29 } & AA & $164.2727 \pm 1.7434^{\mathrm{a}}$ & $146.2273 \pm 2.1370^{\mathrm{a}}$ & $211.2727 \pm 3.5144^{\mathrm{a}}$ & $17.2273 \pm 0.2626^{\mathrm{a}}$ & $497.7660 \pm 18.5661^{\mathrm{a}}$ \\
\hline & $\mathrm{AC}$ & $161.0000 \pm 2.7487^{\mathrm{a}}$ & $141.6667 \pm 2.7436^{\mathrm{a}}$ & $209.1111 \pm 5.7019^{\mathrm{a}}$ & $17.1111 \pm 0.2605^{\mathrm{a}}$ & $477.8839 \pm 26.1093^{\mathrm{a}}$ \\
\hline & $\mathrm{BC}$ & $163.6667 \pm 2.6510^{\mathrm{a}}$ & $146.0000 \pm 4.0276^{\mathrm{a}}$ & $212.1111 \pm 5.5987^{\mathrm{a}}$ & $17.4444 \pm 0.4746^{\mathrm{a}}$ & $500.3969 \pm 28.4474^{\mathrm{a}}$ \\
\hline \multirow[t]{2}{*}{ YWLL36 } & $\mathrm{BD}$ & $156.0000 \pm 9.0000^{\mathrm{a}}$ & $137.5000 \pm 8.5000^{\mathrm{a}}$ & $202.5000 \pm 13.5000^{\mathrm{b}}$ & $16.0000 \pm 0.0000^{\mathrm{a}}$ & $444.7873 \pm 64.0855^{\mathrm{a}}$ \\
\hline & $\mathrm{CE}$ & $163.7895 \pm 1.2639^{\mathrm{a}}$ & $145.5526 \pm 1.6207^{\mathrm{a}}$ & $211.4211 \pm 2.6445^{\mathrm{a}}$ & $17.3158 \pm 0.1889^{\mathrm{a}}$ & $496.4685 \pm 13.5022^{\mathrm{a}}$ \\
\hline \multirow[t]{4}{*}{ YWLL44 } & $\mathrm{AC}$ & $164.0000 \pm 5.0000^{\mathrm{a}}$ & $144.0000 \pm 10.0000^{\mathrm{a}}$ & $205.5000 \pm 5.5000^{b}$ & $16.5000 \pm 0.5000^{\mathrm{b}}$ & $467.9481 \pm 43.5104^{\mathrm{b}}$ \\
\hline & $\mathrm{BD}$ & $162.5714 \pm 1.8510^{\mathrm{a}}$ & $144.6190 \pm 2.2860^{\mathrm{a}}$ & $208.4762 \pm 3.2038^{b}$ & $17.0476 \pm 0.2233^{\mathrm{ab}}$ & $482.9272 \pm 16.3426^{\mathrm{b}}$ \\
\hline & $\mathrm{BE}$ & $162.5000 \pm 1.5468^{\mathrm{a}}$ & $144.6429 \pm 2.4798^{\mathrm{a}}$ & $212.5714 \pm 4.4987^{\mathrm{ab}}$ & $17.4286 \pm 0.2911^{\mathrm{ab}}$ & $498.0232 \pm 21.9750^{\mathrm{b}}$ \\
\hline & $\mathrm{CE}$ & $173.0000 \pm 7.0237^{\mathrm{a}}$ & $152.0000 \pm 7.0000^{\mathrm{a}}$ & $224.6667 \pm 15.8359^{\mathrm{a}}$ & $18.3333 \pm 1.3333^{\mathrm{a}}$ & $568.5623 \pm 86.5378^{a}$ \\
\hline \multirow[t]{2}{*}{ VOLP08 } & $\mathrm{AC}$ & $161.0000 \pm 1.8185^{\mathrm{a}}$ & $142.6111 \pm 2.2825^{\mathrm{a}}$ & $206.5556 \pm 3.8866^{\mathrm{a}}$ & $17.0556 \pm 0.2056^{\mathrm{a}}$ & $471.9134 \pm 18.9111^{\mathrm{a}}$ \\
\hline & $\mathrm{BD}$ & $165.3636 \pm 1.6922^{\mathrm{a}}$ & $147.2273 \pm 2.1601^{\mathrm{a}}$ & $214.5909 \pm 3.3183^{\mathrm{a}}$ & $17.4091 \pm 0.2917^{\mathrm{a}}$ & $511.8608 \pm 17.6782^{\mathrm{a}}$ \\
\hline \multirow[t]{2}{*}{ VOLP32 } & $\mathrm{AC}$ & $164.6087 \pm 1.8657^{\mathrm{a}}$ & $145.8261 \pm 2.2419^{\mathrm{a}}$ & $209.4348 \pm 3.3242^{\mathrm{a}}$ & $17.4348 \pm 0.2654^{\mathrm{a}}$ & $490.2826 \pm 17.8966^{\mathrm{a}}$ \\
\hline & $\mathrm{BD}$ & $161.7647 \pm 1.5846^{\mathrm{a}}$ & $144.2353 \pm 2.2630^{\mathrm{a}}$ & $213.0588 \pm 4.1253^{\mathrm{a}}$ & $17.0000 \pm 0.2425^{\mathrm{a}}$ & $498.7577 \pm 19.8198^{\mathrm{a}}$ \\
\hline
\end{tabular}


Association between polymorphism of microsatellite markers and body size traits of bactrian camels

The association between microsatellite marker polymorphism and body size traits of bactrian camels is shown in Table IV. From Table IV, it can be seen that the body length of $\mathrm{AB}$ genotype marked LCA 33 is significantly higher than that of AA genotype $(P<0.05)$, and the chest circumference, tube circumference and body weight of $\mathrm{AB}$ genotype marked LCA33 are significantly higher than those of AA genotype and AC genotype $(P<0.05)$. The chest circumference and body weight of A A genotype marked LCA90 were significantly higher than those of $\mathrm{AC}$ genotype and $\mathrm{BB}$ genotype $(P<0.05)$. The body length of $\mathrm{AB}$ genotype marked CMS36 was significantly higher than that of AA genotype and $\mathrm{BB}$ genotype $(P<0.05)$. The body weight of CE genotype marked YWLL44 was significantly higher than that of AC genotype, $\mathrm{BD}$ genotype and $\mathrm{BE}$ genotype $(P<0.05)$, the tube circumference of $\mathrm{CE}$ genotype marked YWLL44 was significantly higher than that of AC genotype $(P<0.05)$, and the chest circumference of CE genotype marked YWLL44 was significantly higher than that of AC genotype and BD genotype $(P<0.05)$. The other 13 microsatellite markers had no significant effect on the size traits of Sonid bactrian camels $(P>0.05)$. Therefore, microsatellite markers LCA33, CMS36, LCA90 and YWLL44 can be used in assistant selection of body size and weight markers for Sonid bactrian camels. Which was similar to the study results of Wuren et al. (2018a, $2018 b)$. By now, very few studies on bactrian camels association analysis and genetic mapping were reported, which limited the superior alleles exploring and marker assisted selection breeding developing. The 4 microsatellite markers associated with body size or body weight traits in this study would be valuable in improving bactrian camels by molecular polymerization breeding, which could gather all of the superior alleles from 4 microsatellite markers into one individual.

There are more than 280,000 camels in China, of which Inner Mongolia Autonomous Region is the largest, accounting for about $67 \%$ of the total number of camels in China, followed by Xinjiang Uygur Autonomous Region, accounting for about $20 \%$. To protect the genetic diversity of bactrian camel resources in Inner Mongolia Autonomous Region is not only to rationally manage and utilize the existing resources, but also to maintain a certain resource potential for future needs.

\section{ACKNOWLEDGEMENT}

Sincere gratitude goes to the sponsor of Alxa League Science and Technology Project in Inner Mongolia (2012-12).
Statement of conflict of interest

The authors declare there is no conflict of interest.

\section{REFERENCES}

Bai, J.Y., Pang, Y.Z., Qi, Y.X., Zhang, X.H. and Yun, Y.X., 2017. Analysis of the polymorphism of expressed sequence tag-simple sequence repeat in quail. Indian J. Anim. Res., 51: 851-855. https:// doi.org/10.18805/ijar.v0i0f.3803

Bai, J.Y., Pang, Y.Z., Wu, S.J., Yu, M.Q. and Zhang, X.H., 2016a. Analysis of genetic diversity of four quails by microsatellite markers. Indian J. Anim. Res., 50: 1-7. https://doi.org/10.18805/ijar.8429

Bai, J.Y., Pang, Y.Z., Zhang, X.H., Yun, Y.X. and Qi, Y.X., 2016b. Microsatellite analysis of genetic diversity in quail populations from China. Brazilian J. Poult. Sci., 18: 519-524. https://doi. org/10.1590/1806-9061-2015-0101

Bai, J.Y., Pang, Y.Z., Qi, Y.X., Zhang, X.H. and Yun, X.Y., 2016c. Development and application of Est-Ssr markers in quails. Brazilian J. Poult. Sci., 18(Special Issue): 27-32. https://doi. org/10.1590/1806-9061-2015-0124

Bai, J.Y., Yang, Y.B., Wang, Y.Q., Zhang, X.H. and Pang, Y.Z., 2015. Polymorphism analysis of three Chinese indigenous sheep breeds by microsatellite markers. Indian J. Anim. Res., 49: 585-590. https:// doi.org/10.18805/ijar.5566

Bai, L. and Feng, D.Z., 2015. Study on blood physiological and biochemical characteristics of bactrian camel Chines. Anim. Husb. Vet. Med., 47: 103-106.

Banerjee, P., Joshi, J., Upasna, S., Ganai, N. and Vijh, R.K., 2012. Genetic characterisation of two humped camel of India (Camelus bactrianus). Indian J. Anim. Res., 82: 1205-1212.

Evdotchenko, D., Han, Y., Bartenschlager, H., Preuss, S. and Geldermann, H., 2003. New polymorphic microsatellite loci for different camel species. Mol. Ecol. Notes, 3: 431-434. https://doi.org/10.1046/ j.1471-8286.2003.00477.x

Gao, H.W., Wang, J., He, J.X., Chen, L.Y., Jiri, M. and Meng, H., 2009. Analysis on the genetic diversity and evolution in the Camelus bactrianus using SSR Markers. J. Shanghai Jiaotong Univ. (Agric. Sci.), 27: 89-95.

Guo, L.L., Guo, D.L., Zhao, W. and Hou, X.G., 2018. Newly developed SSR markers reveal genetic diversity and geographical clustering in Paeonia suffruticosa based on flower colour. J. Horticul. Sci. Biotechnol., 93: 416-424. https://doi.org/10.1 
080/14620316.2017.1373039

Hedayat-Evrigh, N., Miraei-Ashtiani, S.R., Shahrebabak, M.M., Evrigh, R.K. and Pourasad, K., 2018. Molecular assessment of genetic diversity in dromedaries and bactrian camel using microsatellite markers. J. Agric. Sci. Technol., 20: 1137-1148.

Li, X.L., 2012. Study on meat characteristics of Alxa bactrian camel. Hohhot: a master's degree thesis of Inner Mongolia Agricultural University.

Li, B.B., Jiang, J.F., Fan, X.C., Zhang, Y., Sun, H.S., Zhang, G.H. and Liu, H. 2017. Molecular characterization of chinese grape landraces (Vitis L.) using microsatellite DNA markers. Hortscience, 52: 533-540.

Ni, W.W., Jiang, A., Zhang, J., Ei, G.X. and Huang, Y.F., 2018. Microsatellite marker-based estimation of the genetic diversity of cattle in Chongqing. Indian J. Anim. Res., 52: 1543-1547. https://doi. org/10.18805/ijar.B-887

Prasad, S., Ali, S.A., Banerjee, P., Joshi, J., Sharma, U. and Vijh, R.K., 2014. Genetic characterization of Malvi camel using microsatellite markers. Int. J. biomed. Life Sci., 5: 286-296.

Tian, Y.Z., Nuerbiya, W., Wang, L.J., Wu, W.W., Xu, X.M., Zhang, Y.H., Azhi, T. and Tian, K.C., 2012. Genetic diversity analysis of 6 Xinjiang local bactrian camel. Anim. Husb. Vet. Med., 44: 38-43.

Vijh, R.K., Tantia, M.S. and Mishra, B., 2007.Genetic diversity and differentiation of dromedarian camel of India. Anim. Biotechnol., 18: 81-90. https://doi. org/10.1080/10495390600648741

Wuren, T.D., Bai, J.Y., Ba, L., Zhou, J.W. and Zhung,
W.B., 2018a. Microsatellite polymorphism and its correlation analysis with body size traits in Alashan Gobi bactrian camel. China Anim. Husb. Vet. Med., 45: 1881-1890.

Wuren, T.D., Bai, J.Y., Dao, L.M., Bao, D. and Zhang, Q., 2017. Analysis on the fineness of Alashan bactrian camel. Hubei Agric. Sci., 56: 2913-2915.

Wuren, T.D., Siqin, T.Y. and Bai, J.Y., 2018b. Correlation between microsatellite polymorphism with body size traits of Alashan Desert bactrian camels. Anim. Husb. Vet. Med., 50: 4-9.

Yang, J., Dai, P.F., Zhou, T.H., Huang, Z.H., Feng, L., Su H.L., Liu, Z.L. and Zhao, G.F., 2013. Genetic diversity and structure of wintersweet (Chimonanthus praecox) revealed by EST-SSR markers. Sci. Horticul., 150: 1-10. https://doi. org/10.1016/j.scienta.2012.11.004

Ye, W.L., Wang, F.L., Xie, Z.H., Wang, Y.G., Lin, B. and Wang, J.L., 2014a. Electron microscopic studies of Lumbar Lymph Nodes in bactrian camels (Camelus bactrianus). J. Camel Pract. Res., 21: 191-198. https://doi.org/10.5958/2277-8934.2014.00033.2

Ye, W.L., Xie, Z.H., Wang, F.L., Gen, X., Dong, S. and Wang, J.L., 2014b. Studies on morphology of hypothalamus in bactrian camels (Camelus bactrianus). J. Camel Pract. Res., 21: 103-109. https://doi.org/10.5958/2277-8934.2014.00020.4

Zhang, M.H., Chen, G.L., Zang, C.J., Dong, J., Huang, X.X. and Lu, D.L., 2016. Analysis of physicochemical indexes of junggar bactrian camel milk. China Anim. Husb. Vet. Med., 43: 2628-2633. 